\title{
Impact of Delay and PDR on Video Transmission H.264/SVC in Dense and Sparse Topologies over Ad Hoc Networks
}

\author{
John Petearson Anzola \\ Professor Electronics \\ Engineering \\ University Foundation Los \\ Libertadores \\ Bogota D.C., Colombia
}

\author{
Andres Camilo Jimenez \\ Professor Electronics \\ Engineering \\ University Foundation Los \\ Libertadores \\ Bogota D.C., Colombia
}

\author{
Luis Alejandro Caycedo \\ Professor Electronics \\ Engineering \\ University Foundation Los \\ Libertadores \\ Bogota D.C., Colombia
}

\begin{abstract}
This article focuses on the analysis of the two most widespread protocols of ad hoc network routing: Ad hoc OnDemand Distance Vector (AODV) and Dynamic Source Routing (DSR), both of them popular, scalable, and reactive routing protocols. It analyzes and compares their performance in H.264/SVC video transmission using NS2 simulation and integration with myEvalSVC, analyzing the provision of Quality of Service (QoS) metrics of Delay End to End and Packet Delivery Ratio (PDR), maintaining the network size unchanged but varying the speed of each node. We will observe the behavior of these routing protocols in two types of topology: Dense and Sparse. It seems that the topology significantly influence the provision of QoS in the transmission of H.264/SVC video on a system with a medium access control scheme by using the Distributed Coordination Function (DCF).
\end{abstract}

\section{General Terms}

Ad hoc Networks, Quality of Service

\section{Keywords}

AODV, DSR, H.264/SVC, Dense and Sparse Topology

\section{INTRODUCTION}

Routing protocols play the chief role in communication networks: they are used to establish, correctly and efficiently, a route between a pair of nodes: source-goal; ensuring the timely delivery of messages [1]. Routing protocols in Mobile Ad hoc Networks (MANETs) can be classified into three categories:

-Proactive

- Reactive (On-demand)

- Hybrids

In proactive protocols, the routes to any destination or part of the network are determined on the fly and are maintained through a periodic update of the routing table. Each node has one or more tables in which are stored the hops used to reach any node in the network. The instant that the topology of the network changes, either by adding a node, repositioning, or the rupture of a bond, it is necessary to flood the network message with "Hello" in broadcast mode to upgrade the breaks and routes of all the tables of the nodes involved in communication.
In reactive protocols, the routes are determined at the time that the source requests a route discovery process. Unlike proactive protocols, it is required that reactive protocols look for ways to reach the target node when initiating or communication for any variation in topology; this feature is what classifies them as On-demand protocols since the network resources to avoid unnecessary packet forwarding are periodically optimized. Negative factors in these protocols increase the delay of packets in the network at the time of route discovery.

Hybrid protocols combine the basic properties of proactive and reactive protocols [2]. This paper focuses on a comparative analysis of AODV and DSR protocols.

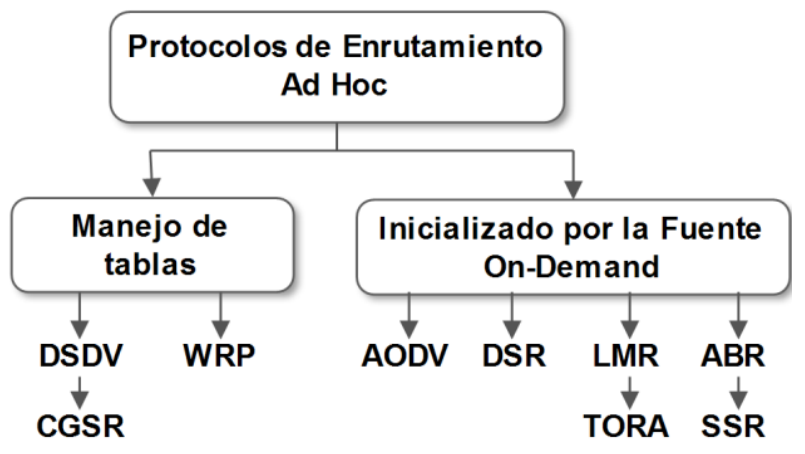

Fig 1: Classification of Ad hoc Routing Protocols [2].

\section{DESCRIPTION OF THE AODV AND DSR PROTOCOLS}

The Ad hoc On-Demand Distance Vector (AODV) protocol was created by Charles E. Perkins [3] as a result of solving the problems encountered in the administration of the routing table of the Destination Sequenced Distance Vector Routing (DSDV). The AODV protocol maintains the principal administration function sequence number and routing tables, but adds the concept of on-demand routing, storing information only on the nodes involved in the communication from end to end.

The improvement over its predecessor lies in its reducing the processing time, memory management and consultation, and in its maintaining a free cache reduction by presenting redundant information in the header (overhead) traffic control. The routing is jump controlled by a vector distance jump, so that no node has full information of the state of the entire 
network: to every possible destination, only the first jump and the distance in hops to the target node are known.

Once a change in the topology occurs (either the entry or exit of a node), the current routing information must be modified and updated with respect to the information of the previous tracks: this requires a timing logic mechanism containing a node ID and a sequence number.

The timer logic is used locally, i.e., there is no absolute timing clock for the entire network. This allows each node to include the state information of the links in the routing table. The logical time of the node that generated the information is included as stored state information and this can have one or more links that do not exist. This mechanism is used in order to give up the outdated information produced by the network topology changes.

\subsection{General operation of the AODV protocol}

Upon discovering a route from a source node to a target node, the first process is carried out by flooding the network with a Route Request (RREQ) packet. Once the RREQ packet reaches the target node or a node that knows the path to the target node, a Route Reply (RREP) packet is generated. This RREP packet has memorized the route source node due to the flooding with the RREQ packet, which was created on the route back. When the RREP packet returns to the source node, the outward route is created. Once the source node receives the RREP packet, the sending of datagrams can begin: they will follow the route generated by the process described above [4].

For the formation of the return route, path requests flood the network and tables point to the source node. If an intermediate node can not answer the route request, it is forwarded, each input and the logical time being stored in the routing tables at the source node. In this process, the route request travels in reverse.

In the formation of the pathway, the RREQ packet is successful if it hits the target node or if a node has memorized the route to the target node. At the time that the RREP packet returns, the tables of intermediate nodes point to the target node, preserving the logical time. If after a certain time no RREP packet has arrived, the user routing tables are deleted. Within each request packet RREQ encapsulates the following information:

- ID of the source node and target.

- Request identifier to control flooding processes.

- Record the logical time at which the target node sends the last known route. For any path not found a zero is returned, signifying that no intermediate node or previous answer knows the route.

- Record the logical time at which the source node sends the request. In the event that the source or any intermediate node presents a topology change, a new node RREQ packet is sent with the information of the new route, replacing the previous information.

-TTL (Time To Live)

RREP response packet whose path is from the target node to the source node, including:

- ID of the source node and the target node.

- Record the time of the target logical node.

-Distance from the source node to the target node.

Route maintenance is done by a detection mechanism for topology changes perceived by alterations in the vicinity, each node maintains information about its neighbors through periodic HELLO messages with a TTL equal to one. Each node maintains information on the active routes so that, when performing a topological change, the routing table is altered reporting an error that requires a new search for active routes. If a neighbor node that has no active participation in any route causes no action disappears.

\subsection{General operation of the DSR protocol}

In using this type of routing, a source node must be able to get the addresses of all the intermediate nodes between the source node and the target node. When sending a packet, the ordered list of intermediate nodes involved in end to end communication must be included in the packet header: this produces a large overhead for the network, affecting its performance. Therefore, one advantage is that intermediate nodes do not require to maintain and update their routing tables, since the routing information is found at the source node, thus avoiding loops routing [5].

The DSR protocol consists of two mechanisms that work together to discover and maintain routes: Route Discovery and Route Maintenance. Route Discovery is the process responsible for obtaining the ID of all the intermediate nodes through which the packets must pass. Route Maintenance is the mechanism by which the source node detects that the route to a given intermediate or final destination can not be used, due to network topology changes. Both processes operate entirely on demand. DSR uses no periodic messages or link status detection. According to the terminology used in DSR, the source node is called the "initiator" and the target node, the "target" [5].

When the initiator has to send a message to a particular target, it performs a route lookup in its "route cache" in order to obtain a previously learned route to the target node. If there is such a route, it is used, otherwise one starts a Route Discovery process to find a new route.

On opening this process, the initiator transmits a Route Request message (RREQ) broadcast type, which is received by all neighboring nodes within the wireless coverage area. Each RREQ post identifies the initiator and the target of the route that it is trying to discover, through the unique identifier of the request (request ID) determined by the initiator node. Additionally, in the process of spreading the message broadcast, there will be collected all the addresses of all the nodes which this particular message has crossed.

This collection of addresses is initialized as an empty list of the initiator node. The request ID is used by the intermediate nodes to determine if a message from the initiator node has been received more than once. If this is the first time a node has received this request, its address is added to the list of nodes that the message has crossed. If a node has received the RREQ message more than once, only the first message is accepted, the rest are discarded. An RREQ message is discarded if the received node address is in the list of nodes it has crossed, i.e., the message has already been received and forwarded by that node.

When the RREQ message has been received by the target node, the target node answers the initiator node with a Route Reply message (RREP) where the collection of the addresses of the intermediate nodes who spoke during the propagation of the RREQ message is included. When the initiator node receives the RREP, it copies the message list of intermediate nodes into the "route cache" as a route for use in sending packets to the target node. 
At this point, the target node has a route to the initiator node to send the RREP message and it does not have to initiate a Route Discovery process, as doing so would trigger the formation of loops.

If a packet is retransmitted, often an intermediate node receives no acknowledgment, and then the node should return an error message to the initiator node, indicating the link from which no response was obtained. If the initiator node has another route on its "route cache" to the target node, it is used, otherwise it must run the Route Discovery process.

An advantage to this protocol is its creating multiple paths to the target node, giving the possibility that each node can control the routes used in packet routing, balancing the network load. DRS is able to work with unidirectional links, which has the disadvantage of increased overhead if the path between the initiator and target nodes is very large or if IPv6 addresses are used.

\section{NODE DENSITY}

The optimum density in MANET networks was analyzed in [6], where the author examines the advantages and disadvantages between connectivity and density of the network nodes, in view of the scalability and mobility of the nodes. The author suggested finding an optimal density to maintain connectivity, as if it were a fixed network. The ratio of the density of the nodes in MANET should be used to measure the transmission range to each node and the network coverage, thus estimating how well connected the network is. In [6], the author defines an area as "dense" when the number of nodes is large and the closeness of a node to another is within the coverage area while maintaining at least two links. Otherwise, the neighborhood density is considered "sparse". However, when determining the density of nodes for a specific area, the connectivity in terms of transmission range is to be considered.

In [8] the density of the network connectivity is determined as follows, defining $\boldsymbol{P}$ as the $k$-connectivity probability, where $\boldsymbol{n}$ is the number of nodes in the network, $\mu$ is determined by Equation (2), $\boldsymbol{\rho}$ is the density, and $\boldsymbol{\pi} \times \boldsymbol{r}_{\mathbf{0}}{ }^{2}$ is the range of transmission coverage area of each node:

$$
\begin{gathered}
P(k-\text { con }) \approx\left(1-e^{-\mu}\right) \cdot n \\
\mu=\rho \times \pi \cdot r_{0}{ }^{2} \\
\rho=\frac{n}{A}
\end{gathered}
$$

Based on the above equations, the author in [6] obtained criteria for determining the size of each topology ("stage"). In Equation (1), the value of $\boldsymbol{k}$ is set to one, so that any network topology is considered as a dense connection with probability $\boldsymbol{P}(\mathbf{1}-\boldsymbol{c o n}) \geq \mathbf{0 . 9 5}$. This likely is a mutually independent way in relation to the connection of the nodes for a particular network area, thus network connectivity is ensured above $95 \%$ [8].

If the density of nodes in the size of each topology or scenario for MANET networks does not guarantee connectivity, this indicates that there is not always a connection between all nodes in the network, since their connection probability $\boldsymbol{P}(\mathbf{1}-\boldsymbol{c o n})<0,95$ is less than $95 \%$, showing that the degree of connectivity in a vicinity is minimal, since the distance between nodes is a jump. This type of topology is called Sparse.

\section{VIDEO H.264/SVC}

An overview of the structure of Scalable Video Coding H.264/SVC, which is composed of scalable multiple layers combining spatial and temporal layers, is presented in this section. The spatial scalability provides support for various screen resolutions, among the most common are 4CIF, CIF and QCIF (Common Intermediate Format) [7], implemented by decomposing the original video into a spatial pyramid.

Temporal scalability is a technique for supporting different frame rates. In SVC, temporal scaling is performed by using a hierarchical prediction structure.

The quality or Signal to Noise Raion (SNR) is also scalable, based on the layers of Coarse Grain Scalable quality (CGS), Medium Grain Scalable quality (MGS), or Fine GranularScalability quality (FGS) [9]. CGS encodes the transform coefficients in a nonscalable way, while FGS arranges the transform coefficients as a stream of embedded bits, allowing truncation at any arbitrary point. In MGS, the coefficients of refinement can be divided into several fragments [10], but can not be truncated at an arbitrary point, increasing the granularity of rate adaptation options. In this research, SVC was used with spatial and temporal scalability using the video "highway_cif.yuv" [11] with CIF $(352 \times 288)$ format at a rate of 30 frames per second.

\section{MODEL OF MOBILITY}

The mobility model used is Random Waypoint with two configuration of work areas (stages): a dense area (1000 $\mathrm{m} \times$ $1000 \mathrm{~m})$ and a Sparse $(1500 \mathrm{~m} \times 1500 \mathrm{~m})$ area. Each of these areas consists of 50 nodes moving without dead times and with varying speeds $(1,2,3,4,5,6,7,8,9,10,11,12,13,14$, 15) $\mathrm{m} / \mathrm{s}$

\subsection{Traffic Model}

The traffic pattern model for the experiments described in this article consists of a traffic Constant Bit Rate (CBR) as background traffic in a system with medium access control Distributed Coordination Function (DCF) traffic on User Datagram Protocol (UDP). The size of each source packet is 512 bytes with a transmission rate of four packages per second. The number of traffic originating nodes can be 10,20 , 30 , or 40

This traffic belongs to a video trace H.264/SVC where the video "highway_cif.yuv" [12], a CIF $(352 \times 288)$ composed of 2000 frames, at a rate of 30 frames per second, is encoded for a total transmission time of $66.6 \mathrm{~s}$ of video. This video was encoded by the myEvalSVC [13] tool, which generates a trace support in an NS2 simulator for a packet transmission rate of 1480 bytes.

\section{PERFORMANCE EVALUATION METRICS}

Routing protocols for ad hoc networks are typically evaluated using the performance indicators Packet Delivery Ratio (PDR) and Average end-to-end Delay (Delay), which are used to measure the effectiveness of the AODV and the DSR [14] protocols. A brief description of these metrics will now be given. 


\subsection{Packet Delivery Ratio (PDR)}

This is the ratio of the number of data packets sent from the source node to the number of packets received by the target node. This metric shows the reliability of the routing protocol: the higher this ratio, the more reliable the packet delivery in the routing protocol's PDR. This metric is given by

$$
P D R=\frac{P_{r}}{P_{S}}
$$

where:

$P_{r}$ is the total number of packets received by the target node and $P_{S}$ is the total number of packets sent by the source node.

\subsection{Average end-to-end Delay (Delay)}

This is the average time elapsed from the start of a packet transmission from source node to the target node. This metric includes all possible delays presented in the network, caused mostly by buffering processes in the initiation, maintenance, and route discovery, the processing times in the queues of the intermediate nodes, packet retransmission delays depending on whether the type of traffic is TCP, the channel access time, and the overhead, among others. The delay is given by

$$
\text { Delay }=T_{r}-T_{s}
$$

where:

$T_{r}$ is the time when a packet is received at the target node and $T_{S}$ is the time in which the packet was sent from the source node.

\section{ANALYSIS}

The analysis and the results of the simulations in NS2 are recorded in this section, giving the results graphically, comparing the AODV with the DSR protocol, both with Dense and Sparse topologies. The packet loss in H.264/SVC video transmission is analyzed using the performance metrics PDR and End to End Delay. The evaluation is performed with $10,20,30$ and 40 traffic sources that account for $20 \%, 40 \%$, $60 \%$ and $80 \%$, respectively, of the background traffic for a network composed of 50 nodes.

\subsection{Dense Topology}

Figures 2 and 3 show the performance parameters (End to End Delay and PDR) for the routing protocols AODV and DSR in a Dense topology consisting of 50 nodes in an area of $1000 \mathrm{~m} \times 1000 \mathrm{~m}$. Generally, it can be seen that the AODV protocol performs better for minor traffic sources up to $40 \%$ of background traffic. For more traffic, this protocol has packet losses greater than $50 \%$, an unsuitable rate for H.264/SVC video transmission, because such a high packet loss does not allow streaming decoding of transmissions.

The loss of video frames of H.264/SVC is notorious in AODV and DSR protocols for speeds above $9 \mathrm{~m} / \mathrm{s}$ background traffic generated with 10 nodes (see Figure 3A). In contrast, with the metric End-to-End Delay, the AODV protocol behavior presents a constant speed change and the DSR protocol reacts more efficiently for a background traffic generated by 10 nodes (see Figure 2A). This tendency of the DSR protocol increases as the speed increases the delay between the nodes. The framework does not allow myEvalSVC encoded videos for which the PDR is less than 0.9, indicating that the H.264/SVC video streaming has a loss greater than $10 \%$ of the transmission.
In scenarios with the Dense topology, the AODV protocol presents a consistent trend for each of the traffic sources analyzed (10, 20, 30 and 40 nodes) in contrast to the DSR protocol, whose delay increases with the rate per node. DSR outperforms AODV in low mobility scenarios whose background traffic is less than $40 \%$ of the total traffic across the entire network.
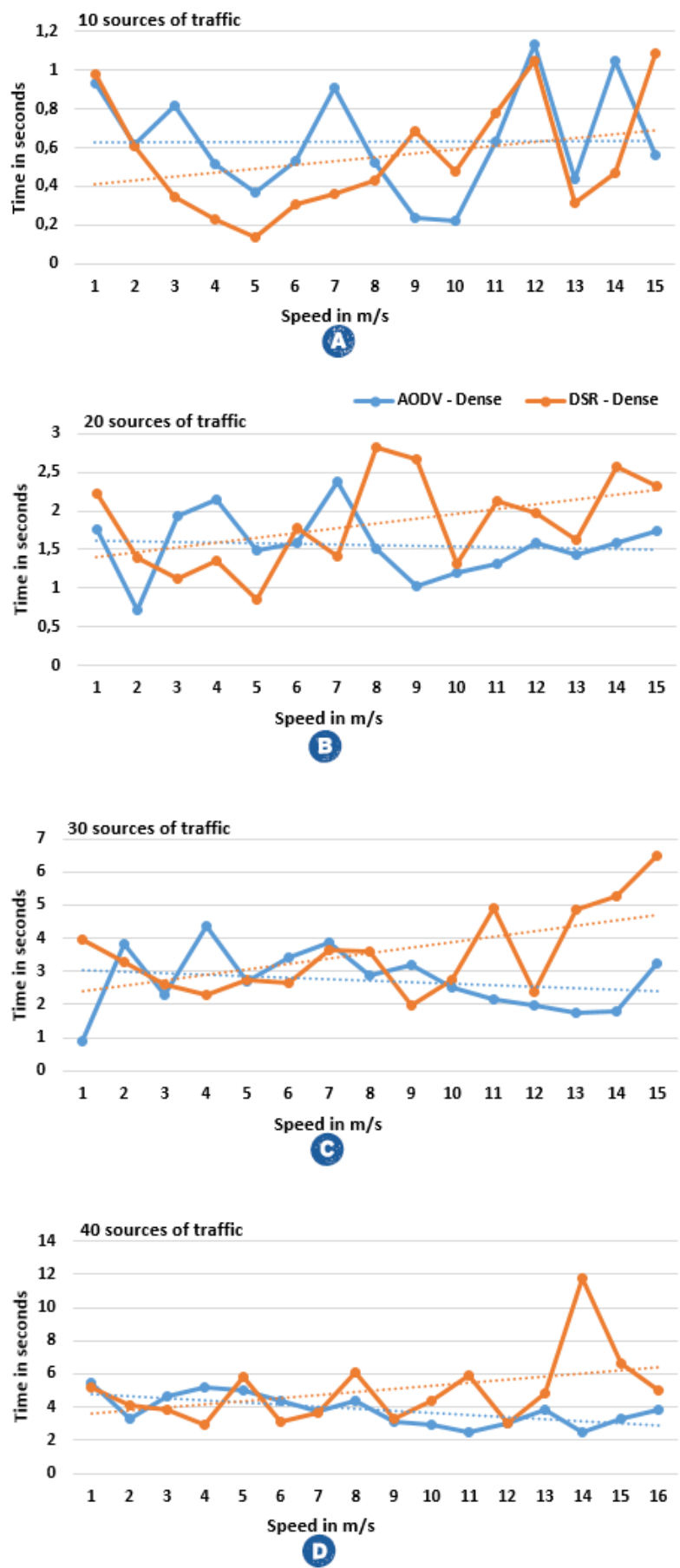

Fig 2: End to End Delay variation. Dense Topology. 

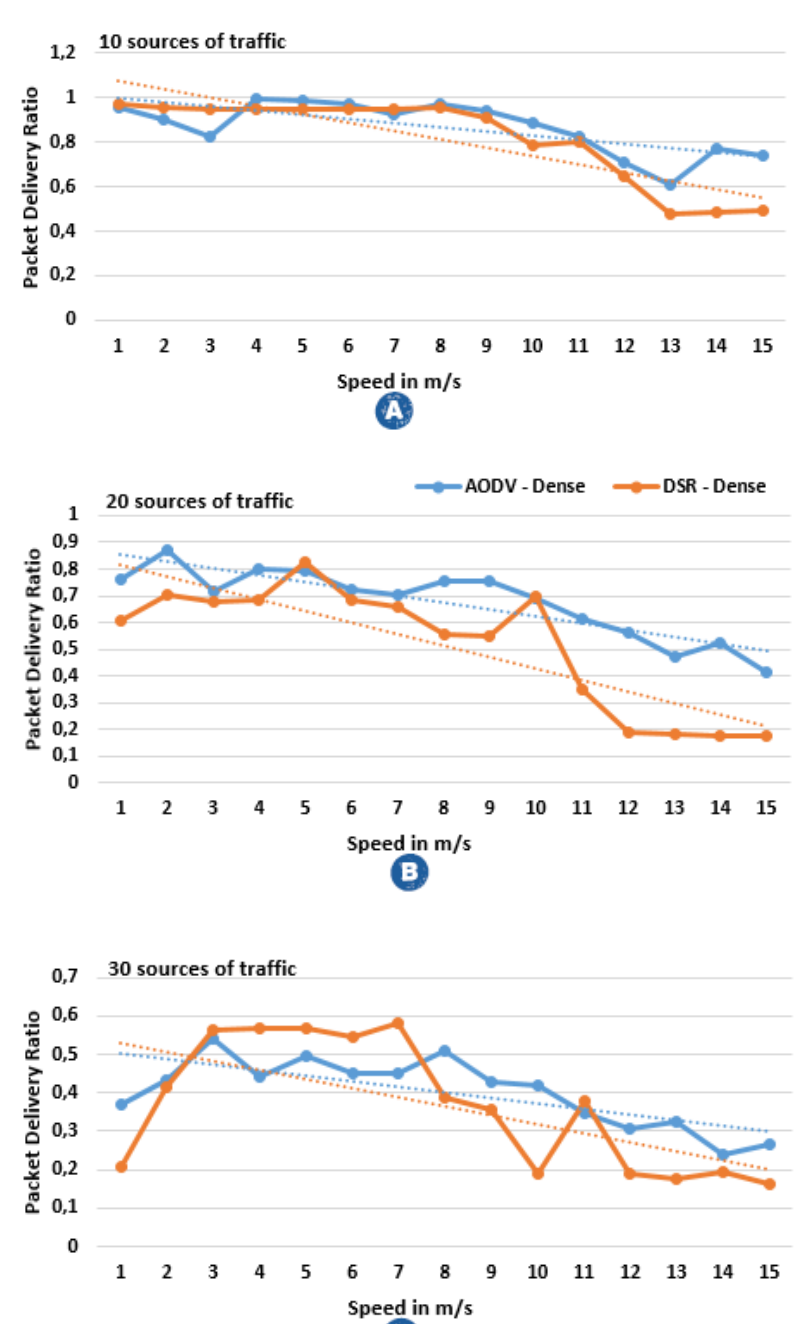

C

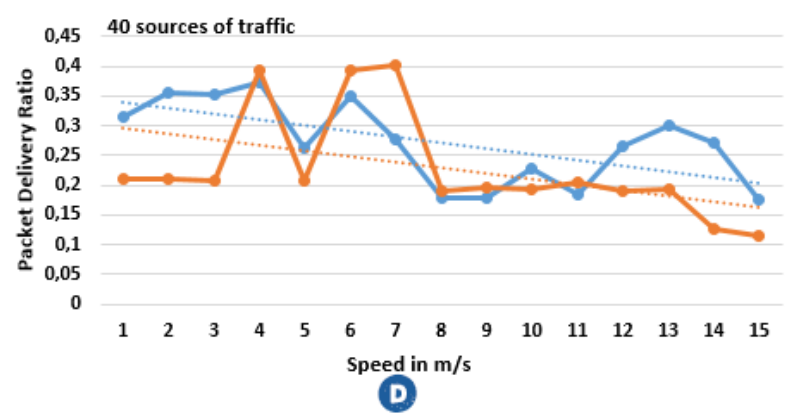

Fig 3: PDR variation. Dense Topology.

In scenarios with the Dense topology, the AODV protocol presents a consistent trend for each of the traffic sources analyzed $(10,20,30$ and 40 nodes) in contrast to the DSR protocol, whose delay increases with the rate per node. DSR outperforms AODV in low mobility scenarios whose background traffic is less than $40 \%$ of the total traffic across the entire network.
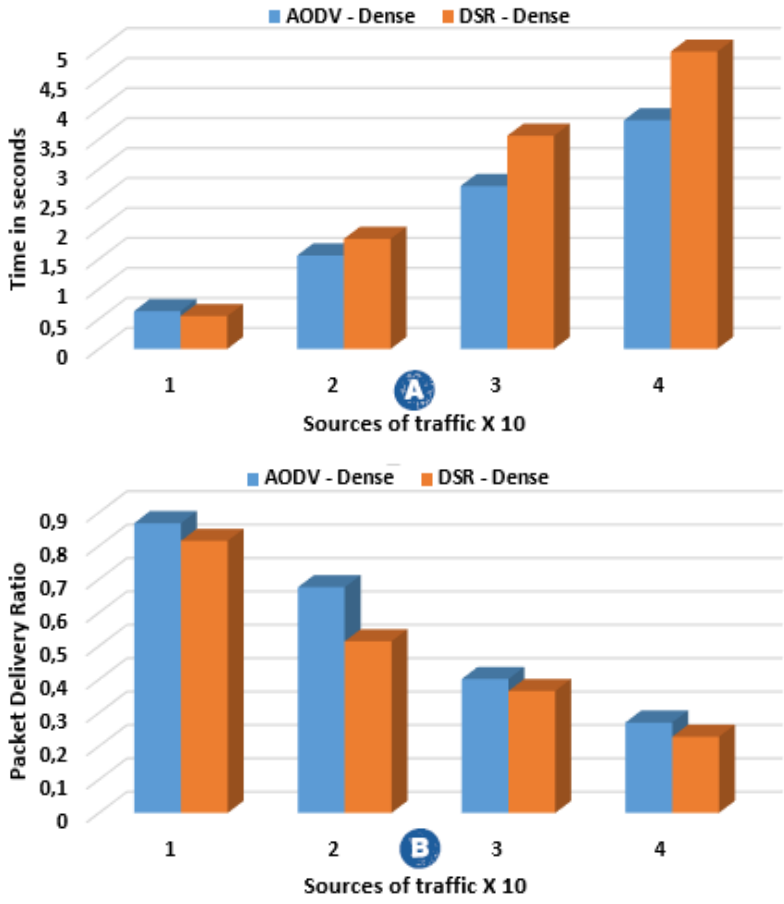

Fig 4: Trend of the AODV and DSR protocols. Dense topology.

\subsection{Sparse Topology}

Figures 5 and 6 show the performance parameters (End to End Delay and PDR) for the routing protocols AODV and DSR in a Sparse topology consisting of 50 nodes in an area of $1500 m \times 1500 m$. In general, it can be seen that the AODV protocol has performs better than DSR for background traffic sources $(10,20,30$ and 40 nodes). In this type of topology, packet losses greater than $30 \%$ are obtained for both the AODV and DSR protocols, which make impossible the H.264/SVC video coding, by the myEvalSVC Framework.
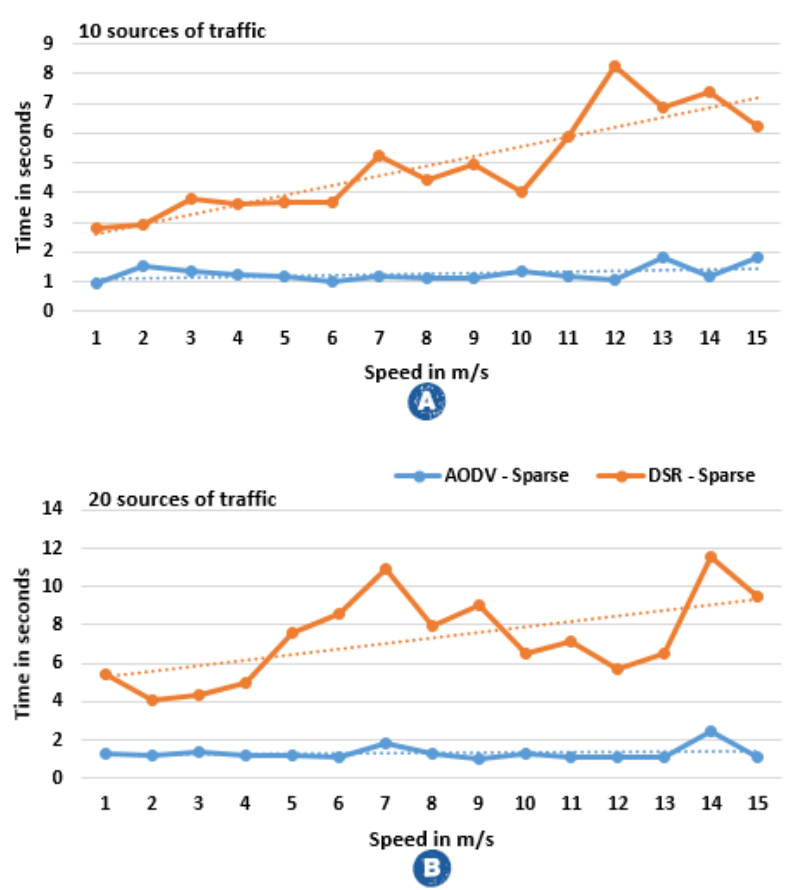

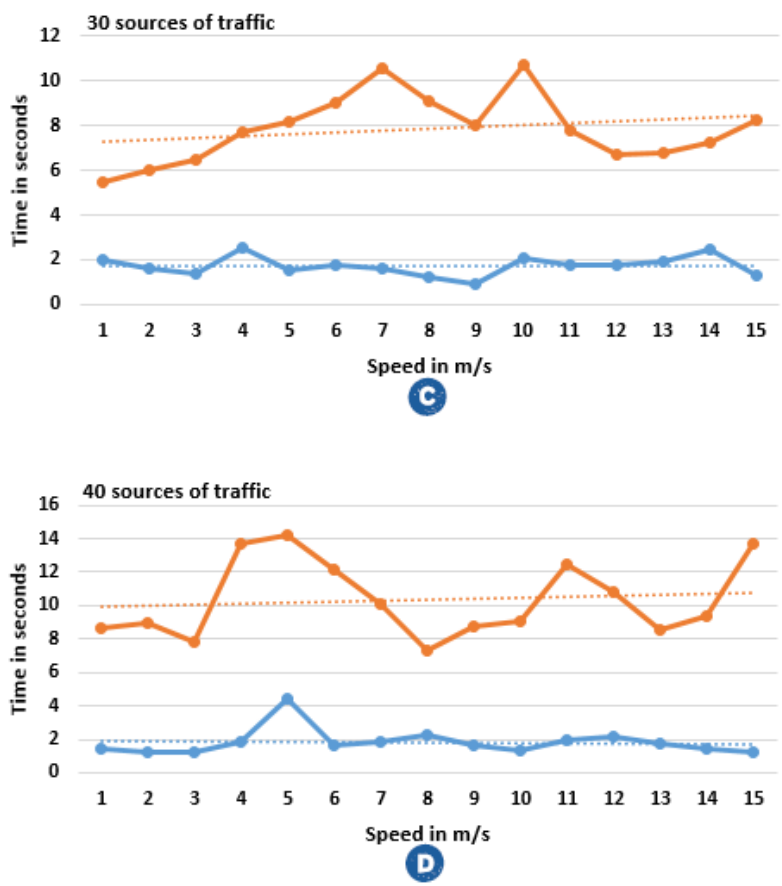

Fig 5: End to End Delay variation. Sparse Topology.
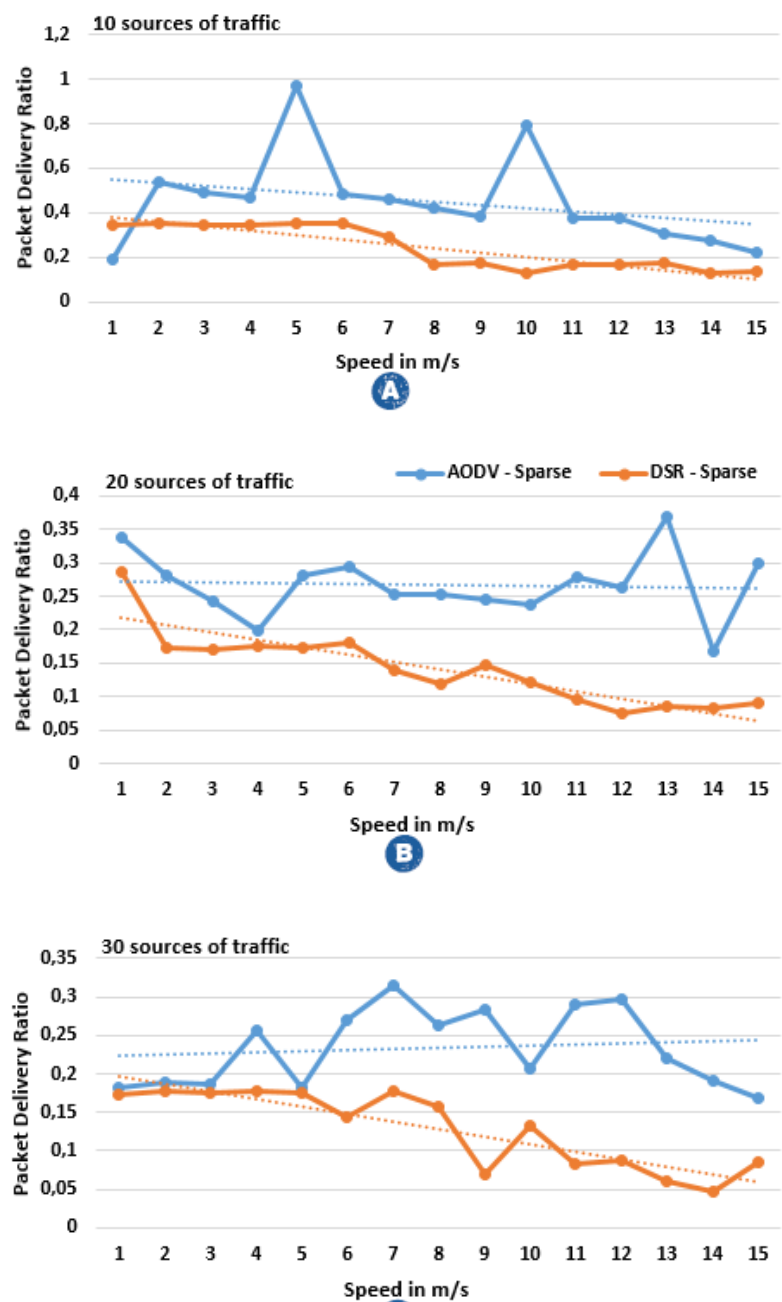

c

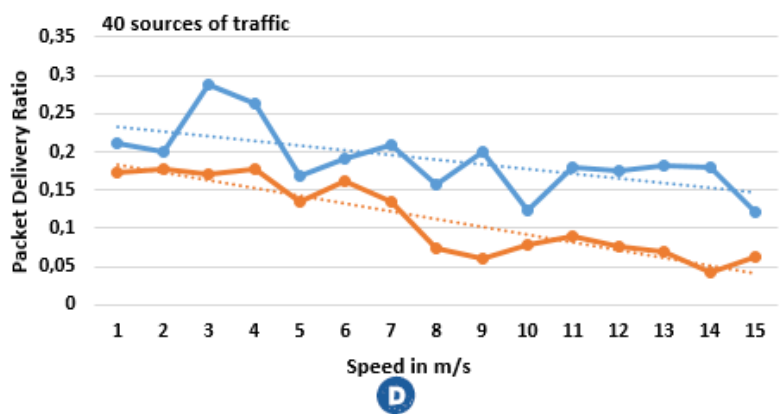

Fig 6: PDR variation. Sparse Topology.

In Sparse topologies, the AODV protocol maintains a constant tendency in its delay with traffic sources of 10, 20, 30 and 40 nodes. The behavior of DSR will directly increase the number of traffic sources and speed. This topology is suitable for the transmission of video streaming H.264/SVC, since it is observed that the PDR is below $50 \%$, so the playback of video in this type of topology is possible.
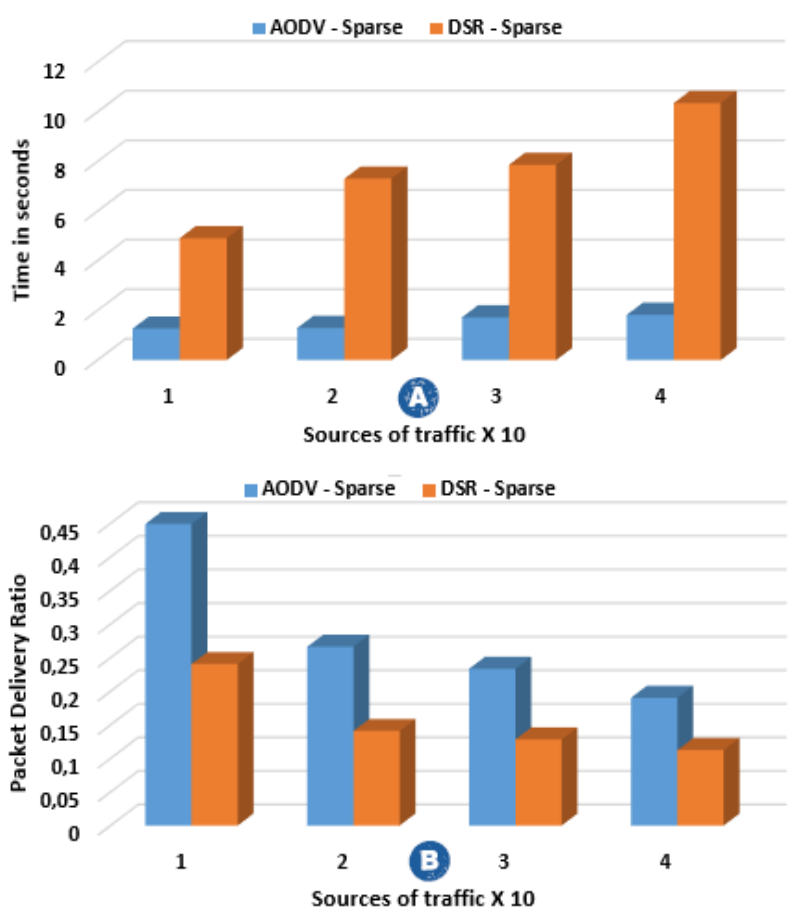

Fig. 7. Trend of the AODV and DSR protocols. Sparse topology.

\subsection{Generalities}

In H.264/SVC video transmission, the AODV protocol has a Dense topologies saturation, increasing the end-to-end delay, compared with the Sparse topologies, whose delay is less and shows a constant trend, but the packet loss in this type of scenario is very high, being above $50 \%$. 


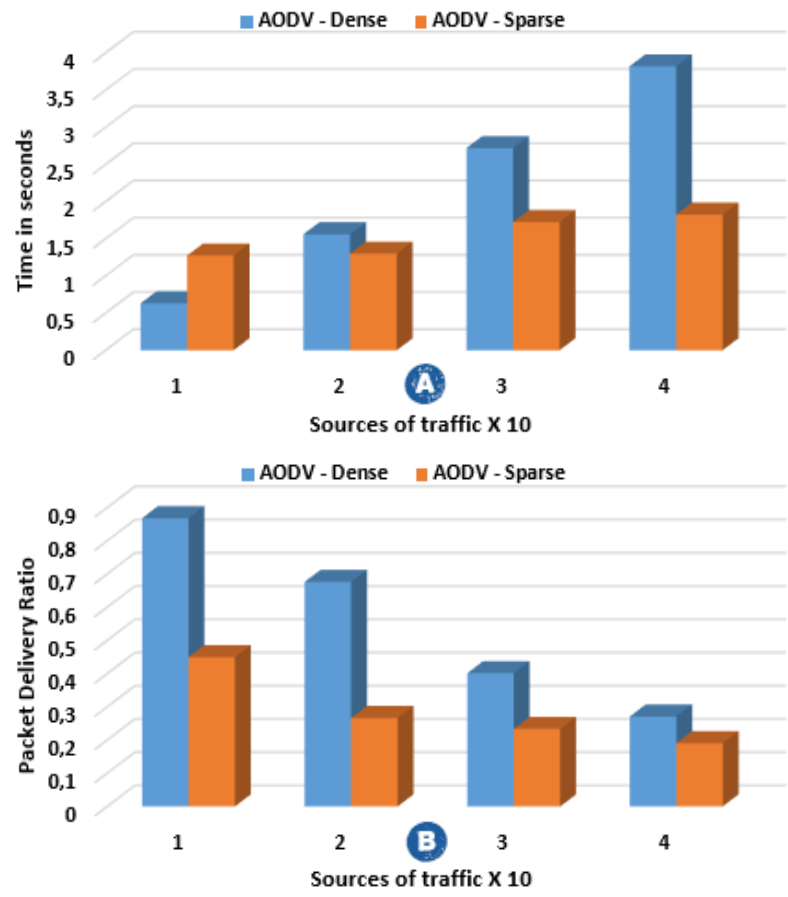

Fig 8: Trend of the AODV protocol.

The DSR protocol has a more stable behavior with respect to changes in the topology from Dense to Sparse, and this is reflected in the PDR, which has a lower packet loss topologies Dense Sparse regarding topology.
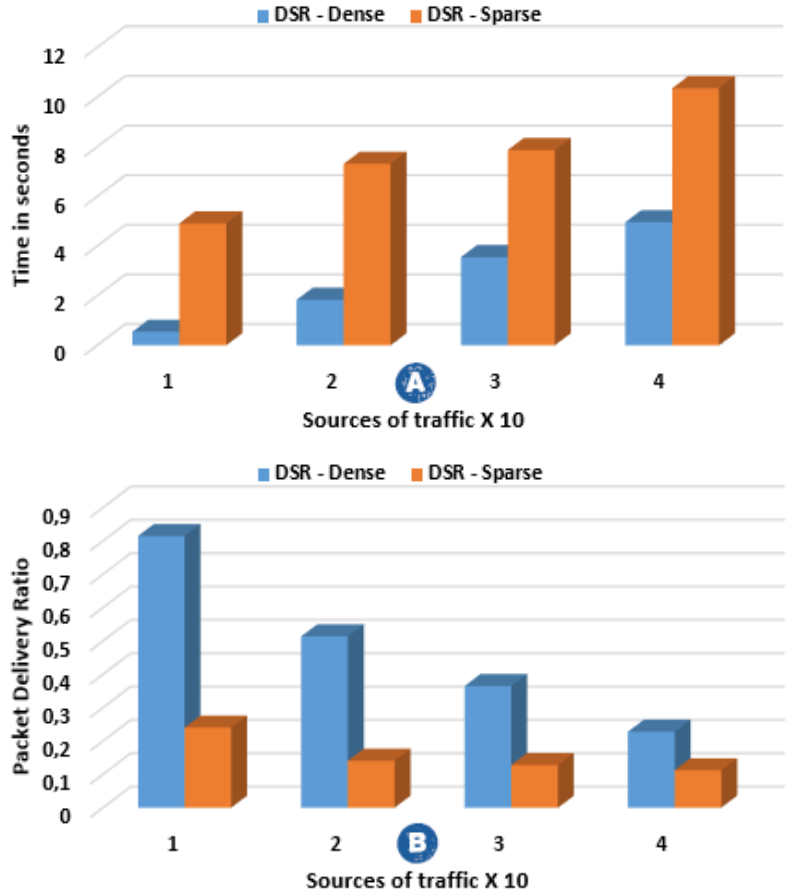

Fig 9: Trend of the DSR protocol.

\section{CONCLUSIONS}

A H.264/SVC video transmission could only be carried out in Dense topologies with lower traffic sources, upt to $10 \%$ of background traffic, for the AODV and DSR protocols with a monitoring mechanism of DCF medium access, noting that the DSR presented slightly better performance in low mobility and background traffic $20 \%$ lower compared with AODV.
The trend of the protocols is that AODV was generally outperformed by DSR in both the Sparse and Dense scenarios: the routing protocol DSR generally has lower End to End Delay and less packet loss, but DSR tends to improve the delay in scenarios with low mobility background traffic.

As for QoS (Quality of Service), the scenarios with the Dense topology significantly exceed the Sparse topologies [6], suggesting that this should be taken into account as a criterion in the analysis of the QoS for MANET networks.

A control mechanism such as the DCF medium access is not recommended for H.264/SVC video transmission, since the video is treated as packages, and conventional data is mixed and processed as background traffic, without any priority. For future experiments, there should be tested the EDCA medium access control mechanism, which specializes in traffic with streaming Audio and Video (dealing with this kind of traffic by using priorities to classify and separate it from background and best effort traffic).

Using DSR is recommended in sensor networks, since these networks have low background traffic and mobility is also low. Furthermore, AODV is recommended for VANETs (Vehicular Ad-Hoc Network), to keep the delay of constant speed changes trend: both protocols are highly scalable, but for a control mechanism such as medium access DCF, the H.264/SVC video transmission is not appropriate.

\section{REFERENCES}

[1] C. Bettstetter. On the connectivity of wireless multihop networks with homogeneous and inhomogeneous range assignment. In Vehicular Technology Conference, 2002. Proceedings. VTC 2002-Fall. 2002 IEEE 56th, volume 3, pages 1706-1710 vol.3, 2002.

[2] Rajesh Deshmukh and Asha Ambhaikar. Article: Performance evaluation of aodv and dsr with reference to network size. International Journal of Computer Applications, 11(8):27-32, December 2010. Published By Foundation of Computer Science.

[3] Video Traces Research Group. Yuv 4:2:0 video sequences. http://trace.eas.asu.edu/yuv/index.html, 72013.

[4] D. Johnson, Y. Hu, and D. Maltz. The Dynamic Source Routing Protocol (DSR) for Mobile Ad Hoc Networks for IPv4. RFC 4728 (Experimental), February 2007.

[5] C. H. Ke. myEvalSVC: an Integrated Simulation Framework for Evaluation of H. 264/SVC Transmission. KSII Transactions on Internet and Information Systems (TIIS), 6(1):377-392, 2012.

[6] James Nightingale, Qi Wang, and Christos Grecos. Realtime video streaming using h.264 scalable video coding (svc) in multihomed mobile networks: a testbed approach, 2011

[7] C. Perkins, E. Royer, and S. Das. RFC 3561 Ad hoc OnDemand Distance Vector (AODV) Routing. Technical report, 2003.

[8] C.E. Perkins. Ad hoc networking. Addison-Wesley networking: mobile computing. Addison-Wesley, 2001.

[9] E.M. Royer, P.M. Melliar-Smith, and L.E. Moser. An analysis of the optimum node density for ad hoc mobile networks. In Communications, 2001. ICC 2001. IEEE International Conference on, volume 3, pages 857-861 vol.3, 2001. 
[10] E.M. Royer and Chai-Keong Toh. A review of current routing protocols for ad hoc mobile wireless networks. Personal Communications, IEEE, 6(2):46-55, 1999.

[11] Heiko Schwarz, Detlev Marpe, and Thomas Wiegand. Overview of the scalable video coding extension of the h.264/avc standard. In IEEE Transactions on Circuits and Systems for Video Technology in Circuits and Systems for Video Technology, pages 1103-1120, 2007.

[12] A. Tuteja, R. Gujral, and S. Thalia. Comparative performance analysis of dsdv, aodv and dsr routing protocols in manet using ns2. In Advances in Computer Engineering (ACE), 2010 International Conference on, pages 330-333, 2010.

[13] ITU (International Telecommunication Union). Recommendation 500-10: Methodology for the subjective assessment of the quality of television pictures. ITU-R Rec. BT.500, 2000.

[14] Zhuo Zhao and Ping Liang. A statistical analysis of h.264/avc fme mode reduction. IEEE Trans. Circuits Syst. Video Techn., 21(1):53-61, 2011 